\title{
Trois espèces de Curculionides nouvelles pour l'île de la Réunion
} (Coleoptera)

Hélène Perrin, Jacques Poussereau, Serge Quilici, Jean-François Voisin

\section{Citer ce document / Cite this document :}

Perrin Hélène, Poussereau Jacques, Quilici Serge, Voisin Jean-François. Trois espèces de Curculionides nouvelles pour l'île de la Réunion (Coleoptera). In: Bulletin de la Société entomologique de France, volume 112 (1), mars 2007. pp. 127-130; https://www.persee.fr/doc/bsef_0037-928x_2007_num_112_1_16409

\section{Ressources associées :}

\section{Curculionides}

Fichier pdf généré le 07/10/2019 


\begin{abstract}
Three species of Curculionids, new for Réunion island (Coleoptera).

Three weevil species have been collected for the first time from Réunion island, Indian Ocean, during the last 15 years. A native to South America widely introduced over the world, Pantomorus cervinus (Boheman, 1840) is spread and now common over a large part of the island. Microlarinus lareynii (Jacquelin du Val, 1852) is only represented by a single specimen collected in 2001 near le Port, and is probably just a short-lived introduction. The case of Baris multivaga Champion, 1914, two specimens of which were collected near le Port in 2003, is more complicated, as this species was described from the Seychelles and is also known from Mauritius, so that it cannot be completely excluded that it is spread on all the region.
\end{abstract}

\title{
Résumé
}

Trois espèces de Curculionides ont été collectées pour la première fois sur l'île de la Réunion pendant ces quinze dernières années. Originaire d'Amérique du Sud et largement introduit de par le monde, Pantomorus cervinus (Boheman, 1840) occupe une grande partie de l'île où il est maintenant commun. Connu par un seul spécimen capturé près du Port en 2001, Microlarinus lareynii (Jacquelin du Val, 1852), espèce méditerranéenne n'est sans doute que le fruit d'une introduction sans lendemain. Baris multivaga Champion, 1914, n'a été trouvé qu'en deux exemplaires dans la région du Port en 2003, mais pose un problème plus complexe, car, décrit des Seychelles, il est aussi présent à l'île Maurice, de sorte que l'on ne peut tout à fait exclure qu'il soit répandu sur toute la région. 


\title{
Trois espèces de Curculionides nouvelles pour l'île de la Réunion (Coleoptera)
}

\author{
par Hélène PERRIN*, Jacques POUSSEREAU**, Serge QUILICI*** \\ et Jean-François VoISIN****
}

*UMS 602, Muséum national d'Histoire naturelle, département Systématique et Évolution (Entomologie), C.P. 50, 57 rue Cuvier, 75005 Paris **193 rue Georges Pompidou, 97430 le Tampon, Réunion ***UMR "Peuplement des Végétaux et Bioagresseurs en Milieu tropical", CIRAD, Réunion, laboratoire d'Écologie terrestre et de Lutte intégrée, 7 chemin de l'IRAT, 97410 Saint-Pierre, Réunion ****USM 305, Muséum national d'Histoire naturelle, C.P. 51, 57 rue Cuvier, 75005 Paris

Résumé. - Trois espèces de Curculionides ont été collectées pour la première fois sur l'île de la Réunion pendant ces quinze demières années. Originaire d'Amérique du Sud et largement introduit de par le monde, Pantomorus cervinus (Boheman, 1840) occupe une grande partie de l'île où il est maintenant commun. Connu par un seul spécimen capturé près du Port en 2001, Microlarinus lareynii (Jacquelin du Val, 1852), espèce méditerranéenne n'est sans doute que le fruit d'une introduction sans lendemain. Baris multivaga Champion, 1914, n'a été trouvé qu'en deux exemplaires dans la région du Port en 2003, mais pose un problème plus complexe, car, décrit des Seychelles, il est aussi présent à l'île Maurice, de sorte que l'on ne peut toụt à fait exclure qu'il soit répandu sur toute la région.

Summary. - Three species of Curculionids, new for Réunion island (Coleoptera). Three weevil species have been collected for the first time from Réunion island, Indian Ocean, during the last 15 years. A native to South America widely introduced over the world, Pantomorus cervinus (Boheman, 1840) is spread and now common over a large part of the island. Microlarinus lareynii (Jacquelin du Val, 1852) is only represented by a single specimen collected in 2001 near le Port, and is probably just a short-lived introduction. The case of Baris multivaga Champion, 1914, two specimens of which were collected near le Port in 2003, is more complicated, as this species was described from the Seychelles and is also known from Mauritius, so that it cannot be completely excluded that it is spread on all the region.

Mots clés. - Coleoptera, Curculionidae, espèces introduites, Réunion.

Les collectes systématiques d'Insectes entreprises à la Réunion depuis plusieurs années ont permis la découverte de plusieurs espèces, nouvelles pour cette île, et même pour la Science, comme on peut s'en rendre compte en lisant la littérature entomologique de ces dernières années. Pour les Curculionides, on peut par exemple citer le travail de WiLLIAMs (2000). Après l'Entiminae Naupactus leucoloma Boheman, 1840, redécrit de la Réunion comme Squamondontus hamoni Richard, 1957 (RICHARD, 1957 ; PERRIN, 2002) et le Molytinae Anchonus interruptus Fåhraeus, 1843, tous deux originaires d'Amérique, déjà signalés (POUSSEREAU \& VOISIN, 2002), nous avons récemment découvert trois autres espèces introduites. Elles font l'objet de la présente note. Les sous-familles sont données d'après ALONSO-ZARAZAGA \& LYAL, 1999.

\section{Pantomorus cervinus (Boheman, 1840) (Entiminae)}

Originaire d'Amérique du Sud, $P$. cervinus a largement été introduit dans le Monde sous des latitudes tropicales et tempérées, et fréquemment dans des îles (Distribution Maps of Pests, $\left.\mathrm{n}^{\circ} 214,1966\right)$. On le trouve même en France métropolitaine : CLERC (1928) le signale sous le nom de Pantomorus godmani (Crotch), HOFFMANN (1950), sous le nom de $P$. fulleri (Horn), puis TEMPÈRE (1977), TEMPĖRE \& PÉRICART (1989), PELLETIER (2005), sous le nom de $P$. cervinus. Comme c'est souvent le cas pour les espèces nuisibles qui se sont répandues dans des zones éloignées de leur région d'origine, ce Charançon a été décrit sous plusieurs noms. Il a déjà été signalé de la Réunion par QUILICI \& LANGLOIS (1992) dans une revue peu accessible, mais GOMY 
Fig. 1. - Distribution de Pantomorus cervinus à la Réunion (d'après les observations de S. Quilici et J. Poussereau).

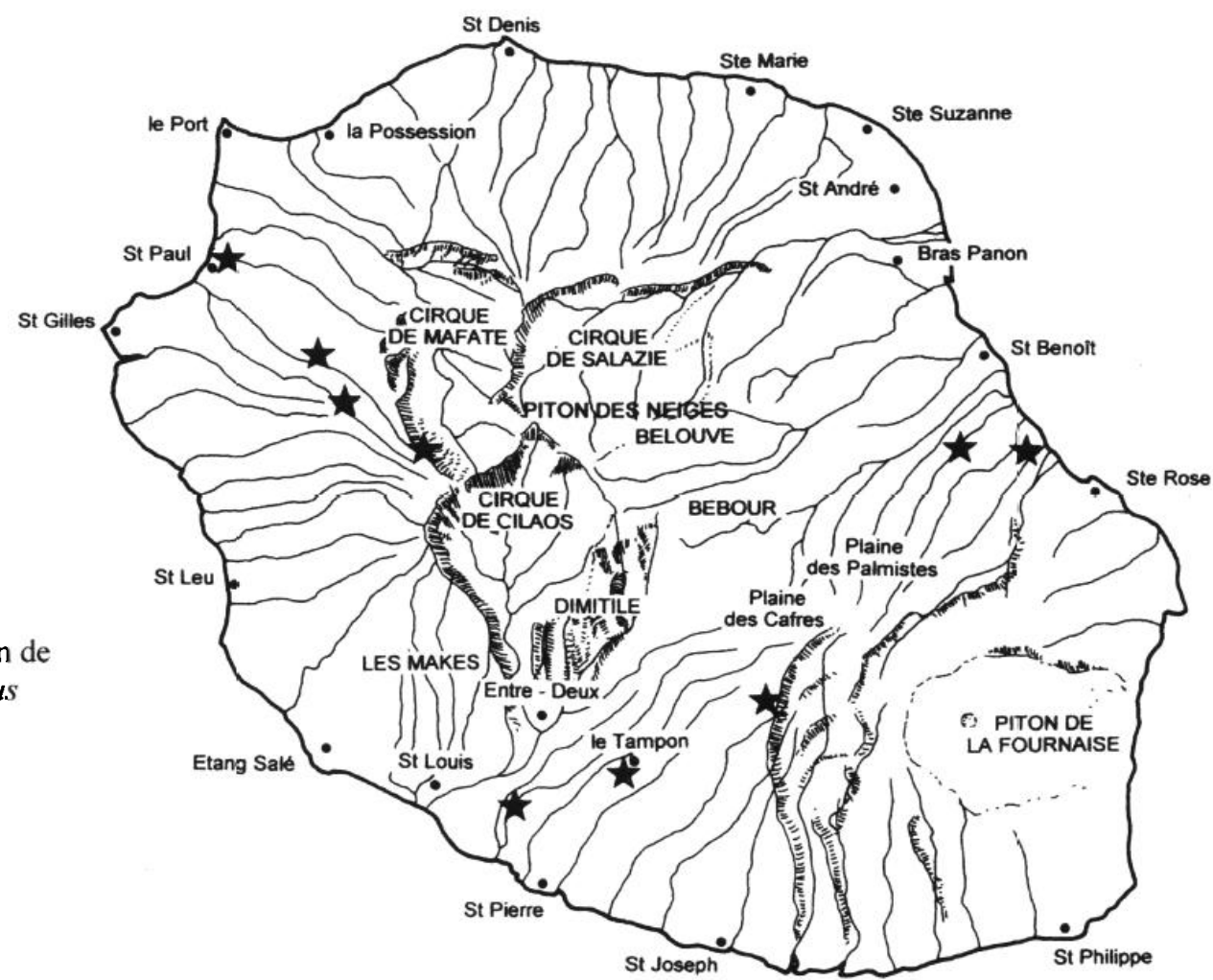

(2000), pourtant fort bien documenté, n'en parle pas. Son introduction à la Réunion est certainement ancienne, car il y est largement répandu (fig. 1) et commun, en particulier dans l'ouest, au-dessus de Saint-Paul, au sud, dans l'Entre-Deux, et au nord-est, près de Saint-Benoît. Extrêmement polyphage, $P$. cervinus s'attaque à toutes sortes de plantes, et en Amérique du Nord et du Sud, par exemple, il peut causer de sérieux dommages à des cultures aussi diverses que les agrumes, les bananes ou le maïs (LANTERI et al., 2002). Son alimentation cst naturcllement tout aussi variée à la Réunion (tabl. D). Sa présence sur diverses plantes qui n'existent pas dans son aire

Tableau I. - Quelques plantes nourricières de Pantomorus cervinus à la Réunion (* $=$ introduit).

\begin{tabular}{llll|} 
Nom scientifique & \multicolumn{2}{c|}{ Nom vernaculaire } & Remarques \\
Lauraceae & Cinnamomum camphora & Camphrier & *cultivé, commun localement \\
Corylaceae & Corylus avellana & Noisetier & *cultivé, moyenne montagne \\
Theaceae & Camellia sinensis & Thé & *cultivé à moyenne altitude \\
Urticaceae & Boehmeria penduliflora & Bois chapelet & *assez répandu dans les Hauts et les Bas ${ }^{1}$ \\
Ericaceae & Rhododendron sp. & Azalée & *Hauts, notamment plaine des Cafres \\
Cunoniaceae & Weinmannia tinctoria & Tan rouge & endémique des Mascareignes, commun \\
Rosaceae & Prunus domestica & Prunier domestique & *cultivé \\
& Prunus persica & Pêcher & *sauvage, basse altitude \\
Fabaceae & Calliandra calothyrsus & Calliandra & * \\
& Leucaena leucocephala & Cassie & * \\
Myrtaceae & Psidium cattleianum & Goyavier & *cultivé, mais envahit les forêts humides \\
Anacardiaceae & Schinus terebinthifolia & Faux poivrier, Baie rose & *répandu en basse et moyenne altitude \\
& & & [espèce envahissante] \\
Geraniaceae & Pelargonium sp. & Géranium & *cultivé dans les Hauts et à moyenne altitude \\
Apocynaceae & Nerium oleander & Laurier-rose & *commun à moyenne et basse altitude \\
Solanaceae & Solanum auriculatum & Bringclicr marron & très répandu [espèce envahissante] \\
& Capsicum frutescens & Petit piment, piment Martin & *cultivé \\
Verbenaceae & Lantana camara & Galabert, Millefleurs & *très répandu [espèce envahissante] \\
Rubiaceae & Anthirea borbonica & Bois d'Osto & dans les Hauts, notamment Roche-Écrite \\
\hline
\end{tabular}

'A la Réunion, on a coutume de désigner sous le nom de "Hauts" les parties élevées de lîle, et sous celui de "Bas" les parties côtières. On appelle "plaines" les zones de hauts plateaux entre Piton des Neiges et Piton de la Fournaise. 


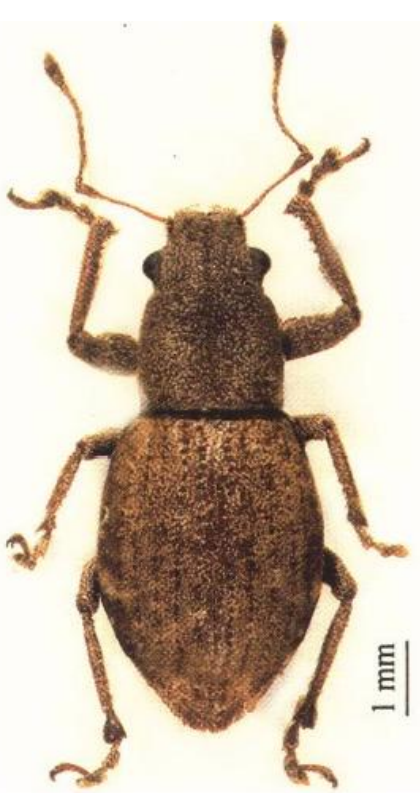

Pantomorus cervinus

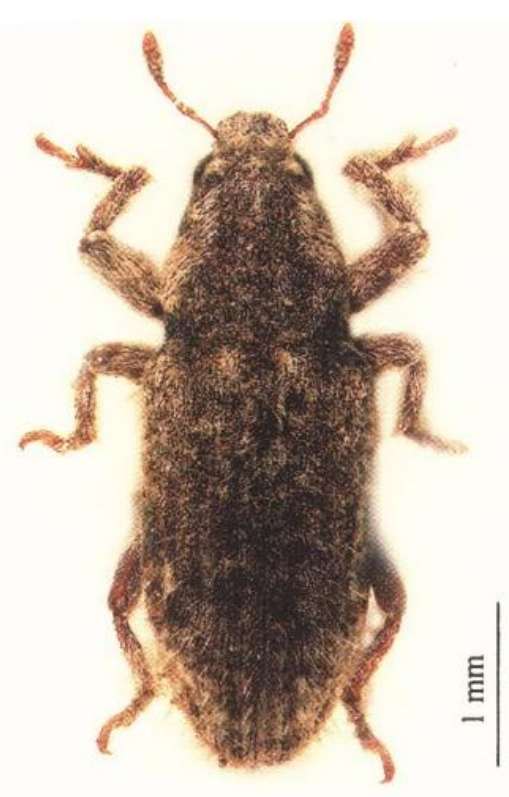

Microlarinus lareynii

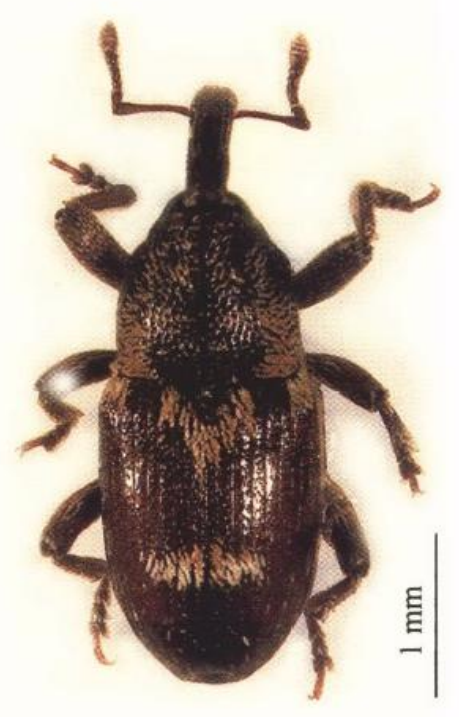

Baris multivaga

de distribution d'origine est remarquable: on le trouve en particulier sur le Laurier-rose (Apocynaceae), qui contient dans toutes ses parties des hétérosides très toxiques (COUPLAN \& STYNER, 1994), et qu'il fréquente en compagnie de Cratopus humeralis Boheman. Ces deux Charançons s'y sont donc adaptés rapidement, en particulier $P$. cervinus.

\section{Microlarinus lareynii (Jacquelin du Val, 1852) (Lixinae)}

Un individu de cette espèce largement répandue dans la région méditerranéenne a été capturé au vol, au Port, le 3 janvier 2001 par l'un de nous (J.P.) La présence de M. lareynii à la Réunion ne peut résulter que d'une introduction, hypothèse confortée par la localité de sa capture dans cette île. C'est une récolte ponctuelle, d'autres captures seraient nécessaires. On connaît assez peu de choses de sa biologie dans sa région d'origine : JACQUELIN DU VAL (1852) précise que ce petit Charançon «vivrait dans les fruits verts du Tribulus terrestris L.» (Zygophyllaceae), information reprise ensuite par d'autres auteurs et développée par HofFMANN (1954, 1962). Son aptitude à se maintenir à la Réunion dépend bien évidemment de son installation sur une plante-hôte, et risque fort de demeurer sans lendemain.

Nota: l'orthographe de l'épithète spécifique de ce Curculionide est bien lareynii, et non lareyniei (PELletier. 2005).

\section{Baris multivaga Champion, 1914 (Baridinae)}

B. multivaga a été décrit des Seychelles, où il avait été récolté dans plusieurs îles lors de la «Percy Sladen trust Expedition» (Champion, 1914). GOMY (2000) indique qu'il se trouve aussi à l'île Maurice. Nous en avons collecté deux spécimens à la Réunion, un sur la plage à Saint-Gilles-les-Bains, le 15 mars 2003, et le second au vol à l'insectarium du Port, le 17 mars de la même année. On peut penser que $B$. multivaga est resté inaperçu à la Réunion du fait de sa petite taille et peut-être de sa rareté, mais le fait qu'il ait été capturé au Port et dans une localité proche de ce dernier, soit dans une région bien prospectée et urbanisée, milite plutôt pour son introduction. Avant de décrire deux espèces de Baris, CHAMPION (op. cit.) précise que dans le genre Baris, très largement distribué dans le monde, quelques espèces ont été accidentellement introduites dans des régions éloignées de leur lieu d'origine. La végétation de la Réunion se rapprochant beaucoup de celle de l'île Maurice, B. multivaga a 
de bonnes chances de s'y maintenir si sa plante nourricière (non connue) s'y trouve. Pour cette espèce, comme pour la précédente d'ailleurs, il est à craindre que les épandages d'insecticides conduits à grande échelle en 2006 pour éradiquer le moustique Aedes albopictus, vecteur du virus du chikungunya, n'aient eu raison de sa petite population.

Il faudrait pouvoir vérifier dans quelques années que ces espèces se sont réellement installées. Nous avons vu que cela semblait être le cas du Pantomorus cervinus, polyphage, signalé déjà en 1992. C'est une hypothèse probable pour Baris multivaga, originaire d'une région voisine, mais c'est beaucoup plus problématique pour Microlarinus lareynii récolté en un seul exemplaire et venant d'une toute autre région.

\section{AUTEURS CITÉS}

Alonso-ZARAZAGa M.A. \& LYAL H.C., 1999. - A world catalogue of families and genera of Curculionoidea (Excepting Scolytidae and Platypodidae). Barcelona: Entomopraxis, $315 \mathrm{p}$.

Champion G.C., 1914. - Coleoptera Curculionidae in The Percy Sladen trust expedition to Indian Ocean in 1905, under the leadership of Mr. J. Stanley Gardiner, M.A., vol. 5, part IV, fasc.19. Transactions of the Linnean Society of London (2), 16 (Zoology): 393-497, 3 pl.

ClerC A., 1928. - A propos de Pantomorus Godmani (Crotch) [Col. Curculionidae]. Bulletin de la Société entomologique de France [1928] : 290-292.

COUPLAN F. \& STYNER E., 1994. - Guide des plantes sauvages comestibles et toxiques. Paris : Delachaux et Niestlé, $415 \mathrm{p}$.

DISTRIBUTION MAPS OF PESTS, Series A (agricultural), $\mathrm{n}^{\circ}$ 214, June 1966. - Pest : Pantomorus cervinus (Boh.) (= Asynonychus godmani Crotch) (Col., Curculionidae) (Fuller's Rose Beetle or Weevil). London : Commonwealth Institute of Entomology. Commonwealth Agricultural Bureaux, $2 \mathrm{p}$.

GOMY Y., 2000. - Nouvelle liste chorologique des coléoptères de l'archipel des Mascareignes. SaintDenis (la Réunion) : Société réunionnaise des Amis du Muséum, $140 \mathrm{p}$.

HoffMAnN A., 1950. - Faune de France, 52. Coléoptères Curculionides ( $1^{\mathrm{re}}$ partie). Paris: Lechevalier, $486 \mathrm{p}$.

- 1954. - Faune de France, 59. Coléoptères Curculionides (2 ${ }^{\mathrm{C}}$ partie). Paris: Lechevalier, 487-1208.

- 1962. - Curculionides de la faune française ( $\Lambda$ dditif à la Faune de France : $3^{\mathrm{e}}$ note). L'Entomologiste, 18: $95-102$.

JACQUELIN DU VAL C., 1852. - Description de deux genres nouveaux et de plusieurs espèces nouvelles de Coléoptères propres à la faune française. Annales de la Société entomologique de France (2) $10: 695-718$.

LanTERI A., Marvaldi A. \& SuARez S, 2002. - Gorgojos de la Argentina y sus plantas huespedes. Publicacion especial de la Sociedad entomologica Argentina, 1 : viii + 1-93, $4 \mathrm{pl}$.

Pelletier J., 2005. - Catalogue des Curculionoidea de France (Coleoptera). Biocosme mésogéen, 2004 $21: 75-147$.

PERRIN H., 2002. - Squamodontus hamoni Richard, 1957, nouveau synonyme de Naupactus leucoloma Boheman, 1840 (Coleoptera, Curculionidae). Revue française d'Entomologie (N.S.), 24 (1) : 33-34.

Poussereau J. \& VOISIN J.-F., 2002. - Découverte de Anchonus interruptus Fåhraeus, 1843, à l'île de la Réunion (Coleoptera, Curculionidae). Bulletin de la Société entomologique de France, 107 (3): 281-283.

QUILICI S. \& LANGLOIS A., 1992. - Biological survey of weevils damaging fruit crops in Réunion Island. Proceedings of the International Society of Citriculture, 39: 1229-1232.

RiCHARD R., 1957. - Coléoptères Curculionidae de La Réunion. Mémoires de l'Institut scientifique de Madagascar (E), 8 : 59-94.

TEMPĖRE G., 1977. - Catalogue des Coléoptères Curculionidae de France. Essai de mise à jour critique ( $2^{\mathrm{e}}$ partie). Entomops, $42: 49-64$.

Tempère G. \& PÉRICART J., 1989. - Faune de France, 74. Coléoplères Curculionidae (4 ${ }^{\mathrm{e}}$ partie). Compléments aux trois volumes d'Adolphe Hoffmann: Corrections, Additions et Répertoire. Paris: Fédération française des Sociétés de Sciences naturelles, $534 \mathrm{p}$.

WILliaMS J.R., 2000. - A revision of the Mascarene weevil genus Syzygops Schönherr (Coleoptera: Curculionidae : Entiminae). Invertebrate Taxonomy, 14:411-432. 